\title{
miRNA-223 as a regulator of inflammation and NLRP3 inflammasome, the main fragments in the puzzle of immunopathogenesis of different inflammatory diseases and COVID-19
}

\author{
Sheyda Houshmandfar ${ }^{1}$ Ali Saeedi-Boroujeni ${ }^{1,2,3}$. Mohammad Rashno ${ }^{1,4}$ - Ali Khodadadi ${ }^{1}$. \\ Mohammad-Reza Mahmoudian-Sani ${ }^{5}$ (i)
}

Received: 16 April 2021 / Accepted: 16 September 2021 / Published online: 30 September 2021

(c) The Author(s), under exclusive licence to Springer-Verlag GmbH Germany, part of Springer Nature 2021

\begin{abstract}
Millions of people around the world are involved with COVID-19 due to infection with SARS-CoV-2. Virological features of SARS-CoV-2, including its genomic sequence, have been identified but the mechanisms governing COVID-19 immunopathogenesis have remained uncertain. miR-223 is a hematopoietic cell-derived miRNA that is implicated in regulating monocyte-macrophage differentiation, neutrophil recruitment, and pro-inflammatory responses. The miR-223 controls inflammation by targeting a variety of factors, including TRAF6, IKK $\alpha$, HSP-70, FOXO1, TLR4, PI3K/AKT, PARP-1, HDAC2, ITGB3, CXCL2, CCL3, IL-6, IFN-I, STMN1, IL-1 $\beta$, IL-18, Caspase-1, NF-kB, and NLRP3. The key role of miR-223 in regulating the inflammatory process and its antioxidant and antiviral role can suggest this miRNA as a potential regulatory factor in the process of COVID-19 immunopathogenesis.
\end{abstract}

Keywords miR-223 $\cdot$ SARS-CoV-2 $\cdot$ COVID-19 $\cdot$ NLRP3 inflammasome $\cdot$ Acute lung injury $\cdot$ Acute respiratory distress syndrome

\section{Introduction}

Besides extensive virology research on SARS-CoV-2, a detailed understanding of virus-host interaction and fundamental, physiological, and immunological processes pave the way for proper diagnosis and design of more effective treatments for infection caused by this virus (Astuti and Ysrafil, 2020). Studies to date have shown that severe

Mohammad-Reza Mahmoudian-Sani

mohamadsani495@gmail.com;

Mahmoudiansani-m@ajums.ac.ir

1 Department of Immunology, Faculty of Medicine, Ahvaz Jundishapur University of Medical Sciences, Ahvaz, Iran

2 Abadan University of Medical Sciences, Abadan, Iran

3 Immunology Today, Universal Scientific Education and Research Network (USERN), Tehran, Iran

4 Cellular \& Molecular Research Center, Medical Basic Sciences Research Institute, Ahvaz Jundishapur University of Medical Sciences, Ahvaz, Iran

5 Thalassemia and Hemoglobinopathy Research Center, Health Research Institute, Ahvaz Jundishapur University of Medical Sciences, Ahvaz, Iran and destructive inflammation is a major cause of death in COVID-19 patients (Gustine and Jones 2020). Cytokine storm refers to a dramatic increase in the levels of tumor necrosis factor- $\alpha$ (TNF- $\alpha$ ), interleukin- 1 beta (IL-1 $\beta$ ), IL-6, IL-8, G-CSF, and GM-CSF cytokines and MCP1, IP10, and MIP1 $\alpha$ inflammatory chemokines, playing a destructive role in inflammatory macrophages and monocytes, which unveils only part of the destructive role of inflammation in COVID-19 (Tang et al. 2020). Topical immune responses are able to effectively clear the virus, although these immune responses themselves in some cases exacerbate the damage caused by the virus. A disorder in the regulation of immune responses occurs in the severe form of COVID-19, similar to Hemophagocytic Lymphohistiocytosis (HLH), sepsis, Kostmann's syndrome, and a variety of immune deficiencies called autoinflammatory syndromes (Lucena et al. 2020). One of the reasons described for the destructive cytokine storm in some patients is the hyperactivation of NLRP3 inflammasome by SARS-CoV-2. The latest structural models of mature SARS-CoV-2 peptides indicated that the virus produces all NLRP3 inflammasome-activating proteins (Freeman and Swartz 2020). In fact, SARS-CoV2 activates the inflammasome and creates a phenomenon called 
pyroptosis as a form of inflammatory programmed cell death pathway associated with the release of IL- $1 \beta$, especially in the epithelial cells of the respiratory tract (Freeman and Swartz 2020). Numerous studies on viral infections reported the inflammasome hyperactivation resulting in destructive and systemic inflammation in patients (Lupfer et al. 2015). The inflammasome function and many key processes implicated in adaptive and innate immune responses are regulated by a group of RNAs called non-coding RNAs (ncRNAs) (Bandyopadhyay et al. 2013). The association of miRNAs with the underlying mechanisms of acute lung injury/acute respiratory distress syndrome (ALI/ARDS) in patients with inflammatory lung injury is not yet fully understood. Among these, the miR-223 prevents inflammation and tissue damage in ARDS. On the other hand, alveolar epithelial type II (ATII) cells are of the main targets of SARS-CoV-2. During mechanical ventilation or injury from a lung infection, neutrophils migrate from the arteries to the site of inflammation of the lungs and damaged tissue, exacerbating the inflammation and destruction (Carcaterra and Caruso 2021). Despite overexpression of miR-223 in myeloid cells, the miR-223 is secreted in neutrophil-derived microvesicles implicated in lung damage and then transferred to ATII cells to participate in lung protection. Upon transfer to ATII cells, the miR-223 inhibits the expression of poly ADP-ribose polymerase 1 (PARP-1) directly by targeting the 3'UTR region to neutralize epithelial inflammation and tissue damage in the ALI. Nanoparticle-mediated delivery of miR-223 to the airway protected the lung in ALI mouse models (Neudecker et al. 2017). It is therefore important that future studies should focus on miR-223 as a promising therapeutic target for the treatment and prevention of ARDS. In this study, we highlight various effects of miR-223 on the inhibition of inflammation in a variety of pathological conditions and discuss the potential role of miR-223 as a diagnostic and therapeutic biomarker in COVID-19.

\section{miRNA, the gene expression regulator, and immune responses}

miRNAs are a non-coding group of 19-23 nucleotide RNAs expressed in multicellular organisms. miRNAs are transcribed in the nucleus of a cell via RNA polymerase II to constitute the initial 1-3 kb long miRNAs. These miRNAs are broken in the nucleus by Drosha Enzyme, turning into a cyclic structure in about 70-100 nucleotides long, called premiRNA. Next, pre-miRNA is transferred from the nucleus to cytoplasm, in which pre-miRNA is divided into mature miRNAs by 18-24 double-stranded oligonucleotides via RNase-III Dicer enzyme. Afterward, this structure affects and regulates different gene expressions by the contribution of other proteins (Filipowicz et al. 2008). According to the studies, microRNAs play an important part in immune cells' plasticity, particularly in differentiating various $\mathrm{T}$ helper cells lymphocytes, such as TH17, TH1, TH2, TH9, TH35, and regulatory T cells (Sethi et al. 2013; Singh et al. 2016). Also, many immune cells need various protein expressions for executive responses, where miRNAs play an important part. Cytokines production regulation and the related signaling pathways, e.g., IFNI, and proinflammatory cytokines playing an important part in pathogens of Coronavirus, are regulated by epigenetic variations like miRNAs (O'connell et al. 2010). According to the studies, the differentiation and function of M1, M2, and Mreg macrophages depend on miRNA expression variations (Zhang et al. 2013).

\section{miR-223, a miRNA derived from hematopoietic cells with a different regulatory role}

The miR-223 is highly conserved during formation and has been described as a modulator of hematopoietic lineage differentiation. miR-223 is a hematopoietic cell-derived miRNA that is implicated in regulating monocyte-macrophage differentiation, neutrophil recruitment, and proinflammatory responses and can be transported to non-myeloid cells via extracellular vesicles or lipoproteins (Roffel et al. 2020; Ye et al. 2018). The key role of miR-223 in regulating the inflammatory process and its antioxidant and antiviral role can suggest this miRNA as a potential regulatory factor in the process of COVID-19 immunopathogenesis. Neutrophils are key components in the development of tissue damage and exacerbation of inflammation in severe cases of COVID-19 (Thierry and Roch 2020). Kruppel-like transcription factor 6 (KLF6) plays a key role in the regulation of inflammatory responses. Many anti-inflammatory and proinflammatory factors ultimately inhibit or exacerbate inflammation by altering KLF6 expression. High expression of KLF6 decreases the expression of miR-223 and conversely, deficiency of KLF6 increases the expression of miR-223 in macrophages (Kim et al. 2019). In recent years, however, various studies have indicated its other roles than a regulator, which will be addressed in different sections.

\section{NLRP3 inflammasome, as an essential multiprotein structure in the innate immune system}

Inflammasomes are cytoplasmic multiprotein complexes that assemble to respond to the Pathogen-Associated Molecular Patterns (PAMPs) and Damage-Associated Molecular Patterns (DAMPs), which are responsible for producing active forms of IL1- $\beta$ and IL-18 cytokines (Martinon et al. 2009). 
Damaged cells, variation in cytosolic ions of cytosolic potassium reduction, microbial products, and increase in extracellular ATP contents cause them to be detected by inflammasomes biomarkers. Besides, activation of inflammasomes leads to a type of cell death along with inflammation in the macrophage and dendritic cells (not in neutrophils and other cells), which is called pyroptosis (Bergsbaken et al. 2009).

The NLRP3 inflammasomes are of the key components of innate immunity, which enhance inflammation by increasing the production of IL-1 $\beta$, IL-18, and gasdermin D, and play a key role in the pathogenesis of many diseases along with destructive inflammation. Thus NLRP3 inflammasome inhibitors, such as flavonoid compounds, can be a potential treatment for severe inflammation, which is the main life-threatening condition in patients with COVID-19 and other types of inflammatory diseases (Saeedi-Boroujeni and Mahmoudian-Sani 2021). As mentioned in the introduction, NLRP3 inflammasome hyperactivity is known as a mechanism that is involved in inflammation and cytokine storm in COVID-19. Accordingly, its accurate regulation is of paramount importance. Along with various factors, the role that different non-coding RNAs, such as LncRNAs and Micro RNA, play in their regulation is indicated (Luo et al. 2021; Tezcan et al. 2019). In this article, the regulatory interaction of miR-223 with NLRP3 inflammasome is investigated and explained.

\section{NLRP3 inflammasome as a potential target for miR-223}

Dysregulation and hyperactivation of the NOD-like receptor family pyrin domain-containing 3 (NLRP3) inflammasome are important factors in the immunopathogenesis of many inflammatory disorders, particularly the severe and lethal form of COVID-19 (Freeman and Swartz 2020). The NLRP3 family is a potential target for miR-223 and plays a key role in viral infections such as hepatitis. The tumor inhibitory role of miR-223 in cancers is associated with the regulation of NLRP3 inflammasome. The miR-223 inhibits hepatocellular carcinoma (HCC) cell proliferation and induces apoptosis by direct targeting of NLRP3. The miR223 also suppresses the production of caspase-1, IL-1 $\beta$, and IL-18 (Wan et al. 2018). The miR-223 is implicated in the regulation of inflammatory immune responses by inhibiting the NLRP3/IL-1 $\beta$ axis in neutrophilic asthma. A study used miR-223-/- mice as a mouse model of OVA/CFA-induced neutrophilic asthma. Exacerbation of neutrophilic inflammation was clearly observed with pathophysiological manifestations and increased production of inflammatory cytokines in miR-223 $3^{-1-}$ mice (Xu et al. 2020). In addition, increased levels of NLRP3 inflammasome and IL-1 $\beta$ were found in miR-223 $3^{-1-}$ mice. Inhibition of NLRP3 inflammasome and depletion of IL-1 $\beta$ clearly prevented the exacerbation of miR-223-deficient-induced neutrophilic inflammation. Overexpression of miR-223 reduces NLRP3 levels and IL-1 $\beta$ secretion and reduces airway inflammation in mice (Xu et al. 2020). Endometritis is an inflammatory disease associated with hyperactive NLRP3 inflammasome and IL-1 $\beta$ (Fusco et al. 2020). The results of a study demonstrated that the miR-223, an inhibitor of NLRP3 inflammasome and inflammatory responses, could be considered as a treatment candidate in dairy cattle with endometritis and other inflammatory diseases. This study well demonstrated the molecular mechanism of miR-223 in reducing the severity of endometritis in dairy cattle. There was a dramatic increase in miR-223 levels in endometritis. NF- $\mathrm{kB}$ activation increases miR-223 expression. Subsequently, miR-223 as a negative regulator reduces the severity of inflammation and the resulting tissue damage by inhibiting NLRP3 inflammasome and IL- $1 \beta$. Consistent with this finding, observations in the mouse model of LPSinduced endometritis showed that inhibition of miR-223 increases the severity of injuries, whereas conversely, the miR-223 overexpression in these mice reduces the severity of inflammation in the uterus of mice (Zhao et al. 2018). A study reported that miR-223, as a myeloid-lineage-specific miRNA, is a key regulator of NLRP3 inflammasome. The miR-223 suppresses NLRP 3 expression by binding to its conserved binding site in the $3^{\prime} \mathrm{UTR}$ of NLRP 3 and thus inhibits NLRP3 inflammasome activation. This study showed that miR-223 expression varies in different types of myeloid cells. Therefore, due to the precise transcriptional control of NLRP3, the function of miR-223 as a regulator of NLRP3 activity is especially important in myeloid cells such as macrophages and neutrophils (Bauernfeind et al. 2012). In a study on the development of myeloid cells, the results interestingly showed that miR-223 is effective in reducing the inflammatory ability of NLRP3 inflammasome. According to this study, the miR-223 expression decreases with differentiation of monocytes into macrophages, while NLRP3 protein increases during this period. However, the miR-223 overexpression prevents the accumulation of NLRP3 protein (Haneklaus et al. 2012). EBV-encoded miRNAs could suppress NLRP3 expression by binding to the miR-223 binding site. Virus-infected B lymphocytes effectively secrete exosomes containing this viral miRNA, thereby inhibiting the activation of NLRP3 inflammasome in other non-virusinfected cells, which can be considered as one of the mechanisms of EBV immune escape. This study is also evidence of the regulatory role of miR-223 in the activation of NLRP3 inflammasome (Haneklaus et al. 2012). Inflammatory bowel disease (IBD) is one of the most prominent disorders with a proven role in the destruction of inflammatory processes and especially in the hyperactivation of NLRP3 inflammasome. In other words, the IBDs can occur as a result of severe and uncontrolled immune responses to microbes in 
the intestinal flora, causing severe tissue damage (Zhen and Zhang 2019). The miR-223 expression is increased in intestinal biopsies of patients with active IBD and in preclinical models of intestinal inflammation. A study found that the miR-223 reduces the severity of IBD by inhibiting NLRP3 inflammasome and reducing IL-1 $\beta$ production (Neudecker et al. 2017c). The miR-223 ${ }^{-/ y}$ mice in the empirical model of colitis showed a much more severe form of the disease with pathophysiological manifestations and intensified production of inflammatory cytokines. On the other hand, in mouse models of NLRP3 3'UTR-deficient as a miR-223 binding region, they also interestingly showed a severe form of IBD, such as miR-223 deficiency in mice with induced colitis. Overexpression of NLRP3 inflammasome and overproduction of IL-1 $\beta$ are important features in the onset of colitis in mice with miR-223 deficiency (Neudecker et al. 2017d). Deletion of CCR2 + inflammatory monocytes and pharmacological inhibition of IL-1 $\beta$ or NLRP3 eliminates the effects of miR-223 deficiency. These findings demonstrated that miR-223 is a key regulatory transcription factor in the regulation of innate immune responses in IBD immunopathogenesis. Finally, nanoparticle-mediated overexpression of miR-223 in colitis-induced mice reduced NLRP3 levels and IL-1 $\beta$ secretion (Neudecker et al. 2017c). Another study found an association between miR-223-3p and NLRP3 inflammatory hyperactivation in obesity for the first time. In subcutaneous fat, a significant correlation was observed between miR-223-3p and miR-223-5p and glucose, NLRP3, and blood lipids (Macartney-Coxson et al. 2020). In another study, the expression levels of miR-223-3p and NLRP3 were measured in glioblastoma tumor tissue. The results showed that miR-223-3p decreased in glioblastoma tissue but NLRP3 increased. Treatment with miR-223-3p reduces the expression of inflammatory chemokines and cytokines such as IL-1 $\beta$, MCP-1, IL-8, and IL-18 and ultimately reduces cancer cell proliferation and migration. Most importantly, the findings of this study showed that the effects of miR-223-3p could be reduced by overexpression of NLRP3, as one of the target genes of miR-223-3p (Ding et al. 2018). In the pathogenesis of alveolar macrophagemediated inflammatory responses, the secretion of miR223/142-containing microvesicles is sharply increased and detected in bronchoalveolar lavage fluid (BALF) and serum. In addition, intracellular miR-223/142 repair and replacement by vesicle delivery inhibit macrophage activation and pneumonia by inhibiting NLRP3 inflammasome activation (Zhang et al. 2019). Studies have shown that SARS-CoV2 could activate inflammasome (Ratajczak et al. 2020). On the other hand, the higher mortality rate of obese patients with COVID-19 is due to the presence of meta-inflammation and NLRP3 inflammasome hyperactivation (Berg and Velde 2020). The function of miR-223 in the regulation of NLRP3 inflammasome activity described in various diseases well demonstrates that this miRNA can play a key role in the immunopathogenesis of COVID-19.

\section{Association of miR-223 with inflammatory lung disease}

Intercellular transfer of miR-223 from neutrophils to pulmonary epithelial cells may reduce ALI by suppressing PARP-1. Overexpression of miR-223 in the lungs protected the mice from ALI induced by mechanical ventilation or by infection with Staphylococcus aureus. Studies of miR-223 gene targets demonstrated that the suppression of PARP-1 by miR-223 is involved in the attenuation of pneumonia (Fig. 1) (Neudecker et al. 2017). Chronic obstructive pulmonary disease (COPD) refers to a chronic disease characterized by airway obstruction and inflammation (Rabe and Watz 2017). Recent findings highlight the importance of miRNAs in the regulation of pathological processes in asthma and COPD (Mohamed et al. 2019). The miR-223 is one of the miRNAs thought to be implicated in the COPD because of changes in its expression level in asthma and COPD (Roffel et al. 2020b). The miR-223 overexpression was observed in mice exposed to cigarette smoke and in emphysematous lung tissue from LPS-treated mice (Leuenberger et al. 2016). In addition, a significant negative relationship was found in miR-223 and HDAC2 expression between two independent cohorts of COPD patients. These findings indicate that the miR-223 is the most common miRNA in COPD, which controls the expression and activity of HDAC2 in lung cells, probably altering the chemokine expression profile. This suggests a new pathogenic association between unregulated miRNA expression and epigenetic activity in COPD. HDAC2 is directly targeted by miR-223 (Fig. 1) whose level is induced by IL- $1 \beta$ and TNF $\alpha$. The miR-223 controls fractalkine expression by targeting HDAC2 (Leuenberger et al. 2016). Restoring miR-223 expression in the lungs of rats with monocrotaline-induced pulmonary arterial hypertension $(\mathrm{PAH})$ reverses $\mathrm{PAH}$ and has beneficial effects on vascular remodeling, pulmonary resistance, right ventricular hypertrophy, and rat survival. Downregulation of miR-223 in PAH plays a key function in several pathways involved in the disease (Meloche et al. 2015). A study examined the function of miR-223-3p and ITGB3 in PAH. The miR223-3p reduces PAH progression by suppressing ITGB3 expression, providing a new target for clinical treatment (Liu et al. 2019). The PAH is a progressive disorder with a poor prognosis, characterized by medial regenerative damage and severe pulmonary vascular dysfunction. The right ventricle is affected by PAH and can cause events such as hypoxia. Findings from a study documented the primary role of pulmonary miR-223 in the right ventricle 
Fig. 1 The potential role of miR-223 in the COVID-19 linked to the suppression of many different target genes

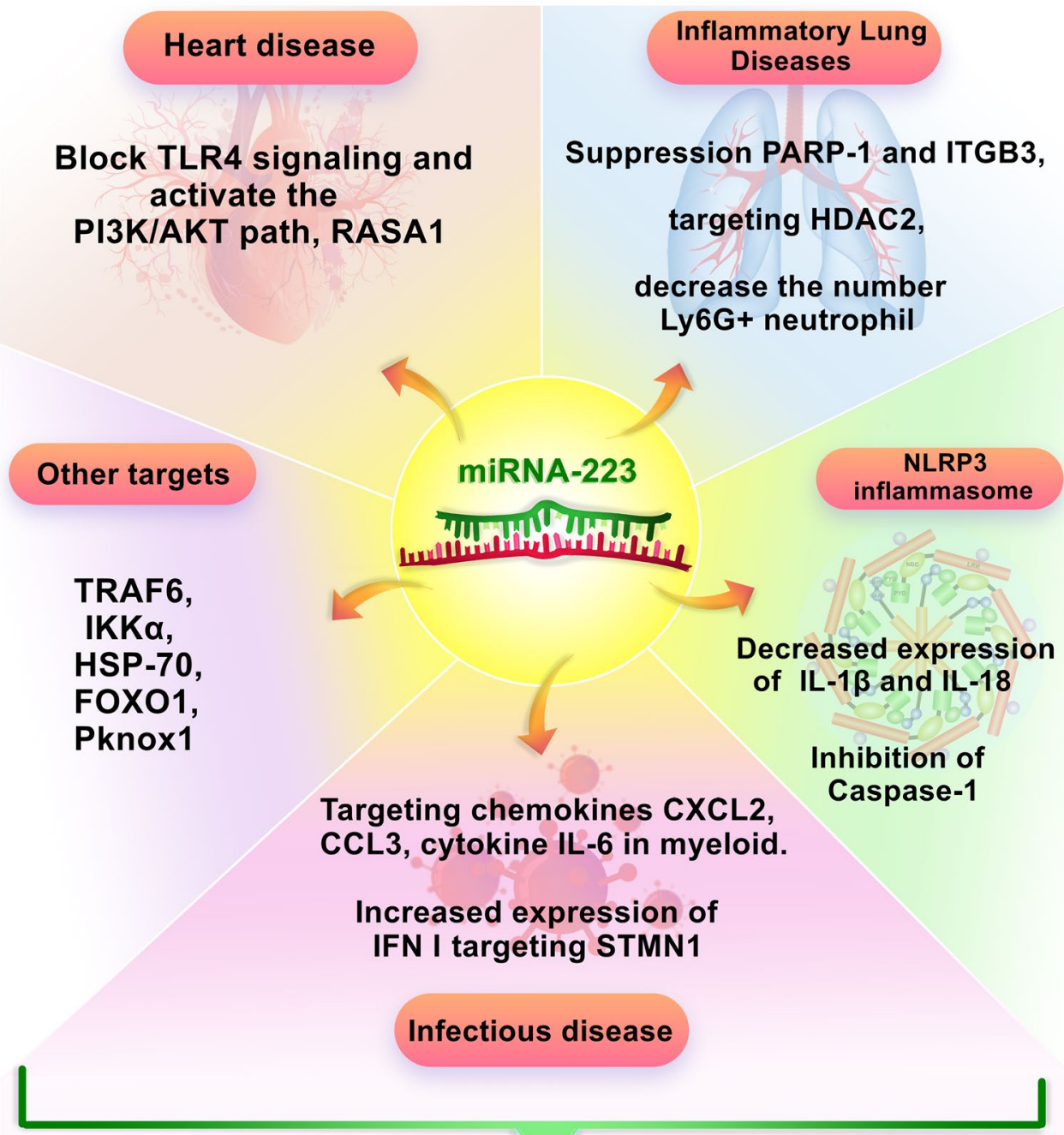

Ameliorate the clinical picture of COVID-19 and insulin-like growth factor-I receptor (IGF-IR) in right heart failure (RHF) caused by pulmonary hypoxia and mechanical overload, probably leading to the development of new treatment strategies aimed at inhibiting the progression of PAH and RHF (Shi et al. 2016). Decreased HDAC2 activity has been reported in COPD patients; Bioinformatics studies have identified miR-223 as a potential suppressor of HDAC2. Functional analysis shows that the miR-223 overexpression decreases HDAC2 expression and activity in Human Pulmonary Artery Endothelial Cells (HPAEC). In contrast, HDAC2 activity was preserved in cells treated with anti-miR-223 (Leuenberger et al. 2016). The results of another study indicate that miR-223 is essential to regulate the pathogenesis of ALI induced by mitochondrial DAMPs. Accordingly, the miR-223 reduces the number of Ly6G + neutrophils and inhibits the activity of the NLRP3 inflammasome to alleviate ALI. The miR223 expression is increased in the lungs of mitochondrial DAMPs-induced mice or ARDS patients following trauma/ transfusion or following the physiological remission of ALI/ARDS. Removal of peripheral blood Ly6G + neutrophils and drug inhibition of the miR-223-NLRP3-IL-1 $\beta$ signaling axis can reduce the severity of mitochondrial DAMPs-induced ALI (Feng et al. 2017). In general, the examination of the role of miR-223 in inflammatory lung diseases clearly raises the need for a detailed study of its role in the development of pneumonia in COVID-19. The cases reviewed in this section are greatly similar to COVID-19 in terms of immunopathogenesis, especially in cases where severe inflammation occurs in the lungs, and so the analysis of miR-223 contains tips for examining its role in COVID-19 by focusing on pneumonia. 


\section{Association of miR-223 with infectious diseases}

During active tuberculosis, myeloid cells invade the lungs, causing and exacerbating local inflammation. Chemokines regulate cellular traffic, but the post-transcriptional events that increase and decrease the expression of these chemotactic factors remain unknown (Domingo-Gonzalez et al. 2016). The miR-223 is increased in the lung and blood parenchyma of patients with tuberculosis as well as rats with tuberculosis. Deletion of miR-223 induced acute lung infection in mice. The lethality of miR-223 $3^{-1-}$ mice was apparently not due to defects in antimycobacterial $\mathrm{T}$ cell responses. Tuberculosis exacerbation in $\mathrm{miR}-223^{-/-}$mice can be partially reversed and attenuated by neutralizing CXCL2, CCL3, and IL-6, by monoclonal antibody depletion of neutrophils, and by genetic deletion of CxCR2 (Xi et al. 2015). In a study, the miR-223 was found to control the lung recruitment of myeloid cells, resulting in neutrophil-induced fatal inflammation. The results of this study demonstrated that miR-223 targets directly the chemokines such as CXCL2 and CCL3 as well as proinflammatory cytokines such as IL-6 in myeloid cells. This study not only demonstrates the essential function for miR-223 in tuberculosis, but also detects novel targets and assigns biofunctions to miR-223. The miR-223 importantly control tuberculosis and other potentially chronic inflammatory diseases through the regulation of leukocyte chemotaxis by chemokines (Dorhoi et al. 2013). Vesicular stomatitis virus (VSV) infection significantly increases miR-223 in rat macrophages. Overexpression of miR-223 was observed to increase the expression level of type I IFN in VSV-infected macrophages. In addition, miR-223 was reported to directly target FOXO3 to regulate type I IFN formation. The VSV-induced type I IFN secretion can increase miR-223 expression, thus forming a positive regulatory loop to produce type I IFN. These results are the first to suggest a new mechanism for regulating the production of type I IFN by miR-223 in the antiviral innate immune response (Chen et al. 2016). Some studies have suggested miR-223 as an antiviral agent. For example, a study found a significant decrease in miR-223 expression levels in human endothelial-like Eahy926 cell line infected with DENV2 virus, whereas increased miR-223 expression effectively inhibited DENV2 proliferation. It was also found that two transcription factors of C/EBP $\alpha$ and E2F1 regulate miR-223 expression after infecting EAhy926 cells with DENV2. The miR-223 as an antiviral agent can be used therapeutically to inhibit DENV (Wu et al. 2014). Interestingly, these studies demonstrate how miR-223 regulates immune responses against pathogens on the one hand by influencing the interferon response as the main antiviral mechanism of host cells, and on the other by regulating leukocyte traffic. It should be noted that severely impaired IFN-mediated antiviral response and impaired leukocyte trafficking are the main causes of dysregulation of immune responses in COVID-19 and severe inflammation.

\section{Association of miR-223 with cardiovascular diseases}

Heart failure is one of the clinical manifestations reported in COVID-19 patients. A review of the role of miR-223 in cardiovascular disease, along with other functions of this miRNA, can help to better understand its potential role in COVID-19 (Greco et al. 2020). The miR-223 expression changes in heart failure after myocardial infarction (MI) in humans; however, the role of miR-223 in MI is unknown. A study aimed to elucidate the role of miR-223 in cardiac fibrosis and reported that miR-223 increases cell proliferation, migration, and differentiation in cardiac fibrosis. The miR-223 may be involved in post-MI cardiac fibrosis in part through targeting RASA1 (Liu et al. 2018). Overexpression of miR-223 may inhibit the progression of atherosclerosis by blocking TLR 4 signaling and conversely by activating PI3K/AKT pathway and therefore poses a promising therapeutic pathway for the treatment of atherosclerosis (Wang et al. 2015). A significant increase in miR-223 level was observed in atherosclerotic lesions in atherosclerotic models of $\mathrm{ApoE}^{-/-}$mice. Overexpression of miR-223 significantly reduces macrophage foam cell formation, lipid accumulation, and inflammatory cytokine production. All of these cases were inversely increased by miR-223 inhibition. The miR-223 negatively regulates TLR4 and NF- $\kappa B$. Pretreatment with a specific NF- $\mathrm{KB}$ inhibitor called pyrrolidine dithiocarbamate (PDTC) severely inhibits the effects induced by silencing miR-223, such as overproduction of inflammatory cytokines and abnormal lipid accumulation. This suggests well that miR-223 induces its effects by affecting NF- $\kappa B$ as a key inflammatory transcription factor. The miR-223 activates the PI3K/AKT signaling pathway. The PI3K/AKT inhibitor, called LY294002, also increases inflammatory cytokine levels and abnormal lipid accumulation by inhibiting miR-223 activity (Wang et al. 2015).

\section{Presence of miR-223 in other diseases}

The miR-223 is abnormally expressed in several diseases such as type 2 diabetes, sepsis, rheumatoid arthritis, viral infections such as HIV-1, and inflammatory disorders (Aziz 2016). Overexpression of miR-125b and miR-223 has been reported in the colonic mucosa of patients with 
ulcerative colitis (UC). A study showed that miR-125b and miR-223 play a role in regulating the inflammatory response during UC through downregulation of the expression of TRAF6, A20, and IKK $\alpha$ to inhibit the inflammation (Valmiki et al. 2019). Another study reported that miR223 could inhibit cell proliferation, increase apoptosis, and inhibit inflammation in retinal ganglion cells (RGCs) by targeting HSP-70 (Ou-Yang et al. 2020). The role of miR233-5p was investigated in the improvement of spinal cord injury (SCI) in rats. Consistent with the findings confirming the key role of miR-223 in the macrophage polarization process, it was interestingly observed that the expression of miR-223-5p increases in M1 microglia, while its expression decreases in M2 microglia. On the other hand, in vivo miR-223 inhibitor effectively inhibits severe inflammation in affected rats. Finally, overexpression of miR-223-5p effectively improves hindlimb locomotion in rats (Guan et al. 2019). The importance of macrophage polarization in the regulation of immune responses, especially in the immunopathogenesis of immune-mediated inflammatory disease (IMID), has been significantly demonstrated recently in various studies. Today, we know that the macrophages not only are a homogeneous population of innate immune cells, but also, we see various types of macrophages with diverse functions depending on varied physiological and pathological conditions (Labonte et al. 2014). One of the main types of macrophage polarization is the existence of two types, classical and alternative. The classic type, also called M1, strongly exacerbates inflammation through hyperproduction of cytokines and inflammatory chemokines. In contrast, the alternative type, also called M2, is more involved in the healing process and inhibition of inflammation and helps to repair tissue by stimulating the induction of fibrosis. The presence of M2 macrophages is higher in the tumor microenvironment due to immunosuppressive conditions, while classically activated macrophages, especially in adipose tissue, increase in inflammatory conditions such as adipose tissue inflammation and systemic insulin resistance (Atri et al. 2018). The miR-223 is involved as a regulator in the macrophage polarization. The miR-223 inhibits the classical pathway and induces alternative macrophages. Pknox 1 is a key regulator of macrophage polarization as a major target for miR-223. Therefore, the miR-223 affects the macrophage polarization and inhibits the induction of classical inflammatory macrophages as an inflammation inhibitor (Zhuang et al. 2012). Examination of changes in miR-223 expression and functional significance of changes in miR-223 expression in fetal organs in the presence of chorioamnionitis showed that embryos with inflammation in the chorioamniotic membrane show overexpression of miR-223 in the thymus, lung, and liver. In addition, FoxO1 is a target of miR-223. Based on these findings, it can be suggested that post-transcriptional regulation of genes by miR-223 is part of the fetal inflammatory response, causing systemic complications in the fetus (Lee et al. 2018). The miR-223-5p/-3p duplexes operate together to inhibit necroptosis caused by ischemia/reperfusion (I/R) in several layers. The pre-miR-223 may be a new candidate for the treatment of ischemic heart disease (Qin et al. 2016).

\section{Conclusion}

COVID-19, especially the severe and lethal form, is associated with severe dysregulation of adaptive and innate immune responses. Lack of effective antiviral responses, such as interferon and exhaustion of cytotoxic T lymphocytes and NK cells on the one hand and systemic inflammation, cytokine storm, and coagulopathy on the other, is the result of this impaired homeostasis and dysregulation of immune system responses. The role of epigenetic modifications in the regulation of immune responses has been demonstrated more than ever in recent years. Among epigenetic modifications, non-coding RNAs such as miRNAs and LncRNAs have been studied more than others. Therefore, any disruption of the immune system response is associated with a variety of pathological conditions from cancer to inflammatory and infectious diseases and with changes in miRNA expression in COVID-19. Numerous studies have reported the role of miR-223 in inhibiting inflammation to prevent tissue damage during infection and other inflammatory diseases. Important targets for miR-223 involved in infection and inflammation are NLRP3, IKK $\alpha$, and NF-KB. The NF- $\kappa B$ and NLRP3 inflammasome are major inflammatory mechanisms, which are inhibited by miR-223. The miR-223 can also target viruses directly. In fact, the miR223 controls inflammation by targeting a variety of factors, including TRAF6, IKK $\alpha$, HSP-70, FOXO1, TLR4, PI3K/ AKT, PARP-1, HDAC2, ITGB3, CXCL2, CCL3, IL-6, IFNI, STMN1, IL-1 $\beta$, IL-18, Caspase-1, NF-KB, and NLRP3. There is compelling evidence of dysregulation in the miR223 expression during pulmonary inflammation, infectious disease, cardiovascular disease, and other inflammatory disorders such as IBD and type II diabetes. Accordingly, the role of mir-223 in regulating inflammation, NLRP3 inflammasome, key transcription factors like NFKB, etc. has been specified in this study. These are among the important points in the immunopathology and of several inflammatory diseases investigated in this study. Concerning the findings and the necessity of a profound understanding of COVID-19 pathogenesis and the pandemic continuation, it is suggested that as an important regulatory factor, the accurate role of mir-223 is investigated in different studies. 
Author contribution $\mathrm{SH}, \mathrm{ASB}$, and MR conceived of the presented idea. MMS wrote the manuscript with support from AK and MR. The authors read and approved the final manuscript. The authors declare that all data were generated in-house and that no paper mill was used.

Data availability The datasets used and/or analyzed during the current study are available from the corresponding author on reasonable request.

\section{Declarations}

Ethics approval and consent to participate Not applicable.

Consent for publication Not applicable.

Competing interests The authors declare no competing interests.

\section{References}

Astuti I, Ysrafil, (2020) Severe acute respiratory syndrome coronavirus 2 (SARS-CoV-2): an overview of viral structure and host response. Diabetes Metab Syndr 14(4):407-12

Atri C, Guerfali FZ, Laouini D (2018) Role of human macrophage polarization in inflammation during infectious diseases. Int J Mol Sci 19(6): 1801

Aziz F (2016) The emerging role of miR-223 as novel potential diagnostic and therapeutic target for inflammatory disorders. Cell Immunol 303:1-6

Bandyopadhyay S, Lane T, Venugopal R, Parthasarathy PT, Cho Y, Galam L et al (2013) MicroRNA-133a-1 regulates inflammasome activation through uncoupling protein-2. Biochem Biophys Res Commun 439(3):407-412

Bauernfeind F, Rieger A, Schildberg FA, Knolle PA, Schmid-Burgk JL, Hornung V (2012) NLRP3 inflammasome activity is negatively controlled by miR-223. J Immunol 189(8):4175-4181

van den Berg DF, te Velde AA (2020) Severe COVID-19: NLRP3 inflammasome dysregulated. Front Immunol 11(1580).

Bergsbaken T, Fink SL, Cookson BT (2009) Pyroptosis: host cell death and inflammation. Nat Rev Microbiol 7(2):99-109

Carcaterra M, Caruso C (2021) Alveolar epithelial cell type II as main target of SARS-CoV-2 virus and COVID-19 development via NF-Kb pathway deregulation: a physio-pathological theory. Med Hypotheses. 146:110412-

Chen L, Song Y, He L, Wan X, Lai L, Dai F et al (2016) MicroRNA-223 promotes type I interferon production in antiviral innate immunity by Targeting forkhead box protein $\mathrm{O} 3$ (FOXO3). J Biol Chem 291(28):14706-14716

de Lucena TMC, da Silva Santos AF, de Lima BR, de Albuquerque Borborema ME, de Azevêdo SJ (2020) Mechanism of inflammatory response in associated comorbidities in COVID-19. Diabetes Metab Syndr 14(4):597-600

Ding Q, Shen L, Nie X, Lu B, Pan X, Su Z et al (2018) MiR-223-3p overexpression inhibits cell proliferation and migration by regulating inflammation-associated cytokines in glioblastomas. Pathol Res Pract 214(9):1330-1339

Domingo-Gonzalez R, Prince O, Cooper A, Khader SA 2016 Cytokines and chemokines in mycobacterium tuberculosis infection. Microbiol Spectr 4(5):https://doi.org/10.1128/microbiolspec. TBTB2-0018-2016.

Dorhoi A, Iannaccone M, Farinacci M, Faé KC, Schreiber J, MouraAlves P et al (2013) MicroRNA-223 controls susceptibility to tuberculosis by regulating lung neutrophil recruitment. J Clin Invest 123(11):4836-4848

Feng Z, Qi S, Zhang Y, Qi Z, Yan L, Zhou J et al (2017) Ly6G+ neutrophil-derived miR-223 inhibits the NLRP3 inflammasome in mitochondrial DAMP-induced acute lung injury. Cell Death Dis. 8(11):e3170

Filipowicz W, Bhattacharyya SN, Sonenberg N (2008) Mechanisms of post-transcriptional regulation by microRNAs: are the answers in sight? Nat Rev Genet 9(2):102-114

Freeman TL, Swartz TH (2020) Targeting the NLRP3 inflammasome in severe COVID-19. Front Immunol 11:1518

Fusco R, Siracusa R, Genovese T, Cuzzocrea S, Di Paola R (2020) Focus on the role of NLRP3 inflammasome in diseases. Int $\mathbf{J}$ Mol Sci 21(12):4223

Greco S, Madè A, Gaetano C, Devaux Y, Emanueli C, Martelli F (2020) Noncoding RNAs implication in cardiovascular diseases in the COVID-19 era. J Transl Med 18(1):408

Guan YZ, Sun C, Wang HL, Xia XL, Lu FZ, Song J et al (2019) MiR-223-5p inhibitor suppresses microglia inflammation and promotes Nrg-1 in rats of spinal cord injury. Eur Rev Med Pharmacol Sci 23(22):9746-9753

Gustine JN, Jones D (2020) Immunopathology of hyperinflammation in COVID-19. The American Journal of Pathology

Haneklaus M, Gerlic M, Kurowska-Stolarska M, Rainey AA, Pich D, McInnes IB et al (2012) Cutting edge: miR-223 and EBV miR-BART15 regulate the NLRP3 inflammasome and IL-1 $\beta$ production. J Immunol 189(8):3795-3799

Kim G-D, Ng HP, Patel N, Mahabeleshwar GH (2019) Kruppel-like factor 6 and miR-223 signaling axis regulates macrophagemediated inflammation. FASEB J 33(10):10902-10915

Labonte AC, Tosello-Trampont AC, Hahn YS (2014) The role of macrophage polarization in infectious and inflammatory diseases. Mol Cells 37(4):275-285

Lee J, Kim CJ, Kim JS, Lee DC, Ahn S, Yoon BH (2018) Increased miR-223 expression in foetal organs is a signature of acute chorioamnionitis with systemic consequences. J Cell Mol Med 22(2):1179-1189

Leuenberger C, Schuoler C, Bye H, Mignan C, Rechsteiner T, Hillinger $\mathrm{S}$ et al (2016) MicroRNA-223 controls the expression of histone deacetylase 2: a novel axis in COPD. J Mol Med (berl) 94(6):725-734

Liu X, Xu Y, Deng Y, Li H (2018) MicroRNA-223 regulates cardiac fibrosis after myocardial infarction by targeting RASA1. Cell Physiol Biochem 46(4):1439-1454

Liu A, Liu Y, Li B, Yang M, Liu Y, Su J (2019) Role of miR-223-3p in pulmonary arterial hypertension via targeting ITGB3 in the ECM pathway. Cell Prolif. 52(2):e12550

Luo D, Liu F, Zhang J, Shao Q, Tao W, Xiao R, et al (2021) Functional crosstalk between long non-coding RNAs and the NLRP3 inflammasome in the regulation of diseases. Molecular Immunology

Lupfer C, Malik A, Kanneganti T-D (2015) Inflammasome control of viral infection. Curr Opin Virol 12:38-46

Macartney-Coxson D, Danielson K, Clapham J, Benton MC, Johnston A, Jones A et al (2020) MicroRNA profiling in adipose before and after weight loss highlights the role of miR-223-3p and the NLRP3 inflammasome. Obesity (silver Spring) 28(3):570-580

Martinon F, Mayor A, Tschopp J (2009) The inflammasomes: guardians of the body. Annu Rev Immunol 27:229-265

Meloche J, Le Guen M, Potus F, Vinck J, Ranchoux B, Johnson I et al (2015) miR-223 reverses experimental pulmonary arterial hypertension. Am J Physiol Cell Physiol 309(6):C363-C372

Mohamed A, Kunda NK, Ross K, Hutcheon GA, Saleem IY (2019) Polymeric nanoparticles for the delivery of miRNA to treat Chronic Obstructive Pulmonary Disease (COPD). Eur J Pharm Biopharm 136:1-8 
Neudecker V, Yuan X, Bowser JL, Eltzschig HK (2017b) MicroRNAs in mucosal inflammation. J Mol Med (berl) 95(9):935-949

Neudecker V, Haneklaus M, Jensen O, Khailova L, Masterson JC, Tye $\mathrm{H}$ et al (2017c) Myeloid-derived miR-223 regulates intestinal inflammation via repression of the NLRP3 inflammasome. J Exp Med 214(6): 1737-1752

Neudecker V, Haneklaus M, Jensen O, Khailova L, Masterson JC, Tye $\mathrm{H}$ et al (2017d) Myeloid-derived miR-223 regulates intestinal inflammation via repression of the NLRP3 inflammasome. J Exp Med 214(6):1737-1752

Neudecker V, Brodsky KS, Clambey ET, Schmidt EP, Packard TA, Davenport B, et al (2017) Neutrophil transfer of miR-223 to lung epithelial cells dampens acute lung injury in mice. Sci Transl Med 9(408)

O'connell RM, Rao DS, Chaudhuri AA, Baltimore D (2010) Physiological and pathological roles for microRNAs in the immune system. Nat Rev Immunol 10(2):111-122

Ou-Yang Y, Liu ZL, Xu CL, Wu JL, Peng J, Peng QH (2020) miR223 induces retinal ganglion cells apoptosis and inflammation via decreasing HSP-70 in vitro and in vivo. J Chem Neuroanat. 104:101747

Qin D, Wang X, Li Y, Yang L, Wang R, Peng J et al (2016) MicroRNA223-5p and $-3 p$ cooperatively suppress necroptosis in ischemic/ reperfused hearts. J Biol Chem 291(38):20247-20259

Rabe KF, Watz H (2017) Chronic obstructive pulmonary disease. Lancet 389(10082): 1931-1940

Ratajczak MZ, Bujko K, Ciechanowicz A, Sielatycka K, Cymer M, Marlicz W, et al (2020) SARS-CoV-2 entry receptor ACE2 is expressed on very small CD45(-) precursors of hematopoietic and endothelial cells and in response to virus spike protein activates the Nlrp3 inflammasome. Stem Cell Rev Rep 1-12.

Roffel MP, Bracke KR, Heijink IH, Maes T (2020) miR-223: a key regulator in the innate immune response in asthma and COPD. Front Med (Lausanne) 7:196-

Roffel MP, Bracke KR, Heijink IH, Maes T (2020b) miR-223: a key regulator in the innate immune response in asthma and COPD. Front Med (lausanne) 7:196

Saeedi-Boroujeni A, Mahmoudian-Sani M-R (2021) Anti-inflammatory potential of Quercetin in COVID-19 treatment. J Inflamm 18(1):3

Sethi A, Kulkarni N, Sonar S, Lal G (2013) Role of miRNAs in CD4 $\mathrm{T}$ cell plasticity during inflammation and tolerance. Frontiers in Genetics 4(8).

Shi L, Kojonazarov B, Elgheznawy A, Popp R, Dahal BK, Böhm M et al (2016) miR-223-IGF-IR signalling in hypoxia- and loadinduced right-ventricular failure: a novel therapeutic approach. Cardiovasc Res 111(3):184-193

Singh Y, Garden OA, Lang F, Cobb BS (2016) Micro RNA s regulate T-cell production of interleukin- 9 and identify hypoxia-inducible factor- $2 \alpha$ as an important regulator of T helper 9 and regulatory T-cell differentiation. Immunology 149(1):74-86

Tang Y, Liu J, Zhang D, Xu Z, Ji J, Wen C (2020) Cytokine storm in COVID-19: the current evidence and treatment strategies. Front Immunol 11:1708-
Tezcan G, Martynova EV, Gilazieva ZE, McIntyre A, Rizvanov AA, Khaiboullina SF (2019) MicroRNA post-transcriptional regulation of the NLRP3 inflammasome in immunopathologies. Front Pharmacol 10:451

Thierry AR, Roch B (2020) Neutrophil extracellular traps and by-products play a key role in COVID-19: pathogenesis, risk factors, and therapy. J Clin Med 9(9):2942

Valmiki S, Ahuja V, Puri N, Paul J (2019) miR-125b and miR-223 contribute to inflammation by targeting the key molecules of $\mathrm{NF \kappa B}$ pathway. Front Med (lausanne) 6:313

Wan L, Yuan X, Liu M, Xue B (2018) miRNA-223-3p regulates NLRP3 to promote apoptosis and inhibit proliferation of hep3B cells. Exp Ther Med 15(3):2429-2435

Wang J, Bai X, Song Q, Fan F, Hu Z, Cheng G et al (2015) miR223 inhibits lipid deposition and inflammation by suppressing toll-like receptor 4 signaling in macrophages. Int J Mol Sci 16(10):24965-24982

Wu N, Gao N, Fan D, Wei J, Zhang J, An J (2014) miR-223 inhibits dengue virus replication by negatively regulating the microtubuledestabilizing protein STMN1 in EAhy926 cells. Microbes Infect 16(11):911-922

Xi X, Zhang C, Han W, Zhao H, Zhang H, Jiao J (2015) MicroRNA-223 is upregulated in active tuberculosis patients and inhibits apoptosis of macrophages by targeting FOXO3. Genet Test Mol Biomarkers 19(12):650-656

Xu W, Wang Y, Ma Y, Yang J (2020) MiR-223 plays a protecting role in neutrophilic asthmatic mice through the inhibition of NLRP3 inflammasome. Respir Res 21(1):116

Ye D, Zhang T, Lou G, Liu Y (2018) Role of miR-223 in the pathophysiology of liver diseases. Exp Mol Med 50(9):128-

Zhang Y, Zhang M, Zhong M, Suo Q, Lv K (2013) Expression profiles of miRNAs in polarized macrophages. Int J Mol Med 31(4):797-802

Zhang D, Lee H, Wang X, Groot M, Sharma L, Dela Cruz CS et al (2019) A potential role of microvesicle-containing miR-223/142 in lung inflammation. Thorax 74(9):865-874

Zhao G, Jiang K, Yang Y, Zhang T, Wu H, Shaukat A et al (2018) The potential therapeutic role of miR-223 in bovine endometritis by targeting the NLRP3 inflammasome. Front Immunol 9:1916

Zhen Y, Zhang H (2019) NLRP3 inflammasome and inflammatory bowel disease. Front Immunol 10:276-

Zhuang G, Meng C, Guo X, Cheruku PS, Shi L, Xu H et al (2012) A novel regulator of macrophage activation: miR-223 in obesity-associated adipose tissue inflammation. Circulation 125(23):2892-2903

Publisher's Note Springer Nature remains neutral with regard to jurisdictional claims in published maps and institutional affiliations. 